\title{
Spin dependent D-brane interactions and scattering amplitudes in matrix theory
}

\author{
J.F. Morales ${ }^{1}$, J.C.Plefka ${ }^{2}$, C.A. Scrucca ${ }^{3}$, M. Serone ${ }^{4}$, and A.K. Waldron ${ }^{5}$ \\ 1 Department of Physics, University "Tor Vergata" of Rome, I-00133 Rome, Italy \\ 2 Albert-Einstein-Institut, Max-Planck-Institut für Gravitationsphysik, D-14473 Potsdam, \\ Germany \\ 3 Sektion Physik, Ludwig Maximilian University of Munich, D-80333 Munich, Germany \\ 4 Department of Mathematics, University of Amsterdam, 1018 TV Amsterdam, The Nether- \\ lands \\ 5 NIKHEF, P.O. Box 41882, 1009 DB Amsterdam, The Netherlands
}

Talk given by M. Serone

\begin{abstract}
Spin interactions between two moving Dp-branes are analyzed using the GreenSchwarz formalism of boundary states. This approach turns out to be extremely efficient to compute all the spin effects related by supersymmetry to the leading $v^{4} / r^{7-p}$ term. All these terms are shown to be scale invariant, supporting a matrix model description of supergravity interactions.

By employing the LSZ reduction formula for matrix theory and the mentioned supersymmetric potential for D0-branes, we compute the t-pole of graviton-graviton and three formthree form scattering in matrix theory. The results are found to be in complete agreement with tree level supergravity in the corresponding kinematical regime and provide, moreover, an explicit map between these degrees of freedom in both theories.
\end{abstract}

\section{Introduction}

The central role played by D-branes [1] in the description of non-perturbative phenomena in string theory has motivated in the last three years the study of their dynamics. In particular, spin dependent long range interactions were first analyzed in [2] for the case of D0-branes through duality arguments. We employ in the following a more direct approach, using the Green-Schwarz boundary state formalism [3], that allows one to study more general brane configurations $[4,5]$. In this approach spin interactions arise by inserting broken supercharges into the partition function of two branes moving past each other. Instead of computing the total partition function corresponding to two moving branes, we can equivalently compute correlation functions of vertex operators, associated to the velocity, on the world-sheet boundary, together with the insertion of broken supercharges that encode spin dependencies.

Consider now the particular case of two parallel Dp-branes in flat space-time ${ }^{1}$. This system leaves unbroken sixteen supercharges meaning, in the Green-Schwarz light-cone formalism, that our action will still admit eight fermionic zero modes $S_{0}^{a}$, in the notation of [6]. Since, as we will see, each insertion of a vertex operator associated to the velocity provides at most two of them, it follows that the potential between parallel

\footnotetext{
${ }^{1}$ More general configurations can be treated in a similar way. See [5] for details.
} 
branes starts with $v^{4}$. On the other hand, the insertion of broken supercharges can allow non-vanishing results for terms with less powers of $v$, providing the lacking fermionic zero modes. Moreover the insertion of supercharges induces polarization-dependent non-minimal couplings between D-branes, i.e. spin-effects. Alternatively, since all these terms are related by supersymmetry [2], it is natural that they are produced by insertion of supercharges.

The correlations we consider, associated to the leading $v^{4-n} / r^{7-p+n}$ terms discussed above will be fixed by zero modes only, since all massive non-BPS bosonic and fermionic string state contributions will always precisely cancel. This implies that these amplitudes, which for large brane separations have a clear interpretation as spin-dependent interactions, due to supersymmetry, present the same functional form at all scales and can be extrapolated to the substringy regime where the dominant degrees of freedom are given by the massless open string states living on the branes. This means in particular that a one-loop matrix theory [7] computation should be able to reproduce long range spin-dependent supergravity interactions. This has been indeed partially shown in [8]. Motivated by the argument above, one could also ask whether matrix theory is able to reproduce supergravity scattering amplitudes. To date, typical matrix theory computations involve the comparison of classical gravity source-probe actions with the background field effective action of super Yang-Mills theory in $(1+0)$ dimensions, evaluated on straight line configurations ${ }^{2}$.

We will be able to go beyond this aproximation by constructing a matrix theory analogue of the Lehmann, Symanzik and Zimmermann (LSZ) reduction formula. In this way we can relate S-matrix elements of asymptotic super-graviton states, whose explicit form has been worked out in [10], to the background field expansion of the matrix theory path integral. Thereafter, all the dynamics will be encoded in the matrix theory effective potential. At the one-loop leading level, however, this potential will precisely coincide with the scale invariant spin dependent D0-brane interactions computed above through the Green-Schwarz boundary state. Combining then this effective potential with the knowledge of the asymptotic super-graviton states in matrix theory, we will be able to extract t-poles of S-matrix elements, in the kinematical configuration with vanishing Kaluza Klein momentum transfer associated to the circle compactification [11, 12]. As particular example, we report here the case of graviton-graviton scattering, showing complete agreement with the corresponding tree level supergravity amplitude ${ }^{3}$.

Throughout the following we work in the $N=2$ sector of the matrix model, so that we are actually considering the Susskind finite $N$ generalisation [13] of the matrix theory conjecture.

\section{Spin effects in the GS boundary state}

D-branes, as world-sheet boundaries, can be described by suitable closed string states, called boundary states, that implement the usual Neumann-Dirichlet boundary conditions, both in the covariant as well as the Green-Schwarz formalism. In the latter

\footnotetext{
${ }^{2}$ See [9] for an exhaustive list of references.

3 The explicit computation for three form-three form scattering, again in complete agreement with supergravity, has been performed in [12].
} 
framework, the boundary state describing a single flat D-brane is defined by the conditions

$$
\left(\partial X^{i}+M_{j}^{i} \bar{\partial} X^{j}\right)|B\rangle=0
$$

and the BPS conditions

$$
\left(Q^{a}+i M_{a \dot{b}} \tilde{Q}^{\dot{b}}\right)|B\rangle=0, \quad\left(Q^{\dot{a}}+i M_{\dot{a} b} \tilde{Q}^{b}\right)|B\rangle=0
$$

where $Q^{a}=\left(2 p^{+}\right)^{1 / 2} \oint d \sigma S^{a}, Q^{\dot{a}}=\left(p^{+}\right)^{-1 / 2} \gamma_{\dot{a} a}^{i} \oint d \sigma \partial X^{i} S^{a}$ are the usual linearly and non-linearly realized light-cone supercharges. The same of course for the right-moving ones. $M_{i j}, M_{a \dot{a}}, M_{\dot{a} a}$ are definite $S O(8)$ matrices $[3,4]$, depending on the dimensionality of the brane ${ }^{4}$. The solution for $|B\rangle$ turns out to be

$$
|B\rangle=\exp \sum_{n>0}\left(\frac{1}{n} M_{i j} \alpha_{-n}^{i} \widetilde{\alpha}_{-n}^{j}-i M_{a \dot{a}} S_{-n}^{a} \widetilde{S}_{-n}^{\dot{a}}\right)\left|B_{0}\right\rangle
$$

$\left|B_{0}\right\rangle$ being the zero mode part,

$$
\left.\left|B_{0}\right\rangle=M_{i j}|i\rangle \widetilde{j}\right\rangle-i M_{\dot{a} b}|\dot{a}\rangle|\widetilde{b}\rangle
$$

In this gauge, the \pm light-cone directions satisfy automatically Dirichlet boundary conditions, meaning that the branes we are dealing with are actually Euclidean branes. The boundary state associated to moving branes is obtained by simply boosting the static one. Although in light-cone gauge this procedure turns out to be problematic, it is possible to overcome this difficulty by identifying one of the $\mathrm{SO}(8)$ transverse directions with the time direction $[4,5]$. Thereafter one deduces the corresponding $S O(1,9)$ expressions and performs a double analytic continuation to the final covariant result.

The configuration space boundary state $|B, \mathbf{x}\rangle$ is given by

$$
|B, \mathbf{x}\rangle=\left(2 \pi \sqrt{\alpha^{\prime}}\right)^{4-p} \int \frac{d^{9-p} q}{(2 \pi)^{9-p}} e^{i \mathbf{q} \cdot \mathbf{x}}|B\rangle \otimes|\mathbf{q}\rangle
$$

with $\left\langle q \mid q^{\prime}\right\rangle=\operatorname{vol}_{p+1}(2 \pi)^{9-p} \delta^{(9-p)}\left(q-q^{\prime}\right)$ and $\operatorname{vol}_{p+1}$ is the space-time volume spanned by the p-brane. In this way the static force between two parallel branes is

$$
\mathcal{A}=\int_{0}^{\infty} d t\left\langle B, \mathbf{x}\left|e^{-2 \pi t \alpha^{\prime} p^{+}\left(P^{-}-i \partial / \partial x^{+}\right)}\right| B, \mathbf{y}\right\rangle
$$

with $P^{-}=\frac{\left(p^{i}\right)^{2}}{p^{+}}+$osc. the light-cone Hamiltonian (the term $i \partial / \partial x^{+}$just implements the $p^{-}$subtraction needed to obtain the effective Hamiltonian in this gauge). From (6) we get

$$
\mathcal{A}=V_{p+1}\left(4 \pi^{2} \alpha^{\prime}\right)^{4-p} \int_{0}^{\infty} d t \int \frac{d^{9-p} q}{(2 \pi)^{9-p}} e^{i \mathbf{q} \cdot(\mathbf{x}-\mathbf{y})} e^{-\pi t \alpha^{\prime} \mathbf{q}^{2}}(8-8) \prod_{n=1}^{\infty} \frac{\left(1-e^{-2 \pi t n}\right)^{8}}{\left(1-e^{-2 \pi t n}\right)^{8}}
$$

\footnotetext{
${ }^{4}$ In writing $M_{a \dot{a}}$ we have implicitly chosen to work in the IIA theory, relevant for the analysis of D-particles.
} 
where the factor $(8-8)$ is due to the trace performed on the zero mode part of the boundary state, eq.(4). Note in particular that massive string contributions precisely cancel from the amplitude.

A generic one-loop $n$-point function of vertex operators $V_{1}, \ldots, V_{n}$ will be then given by

$$
\mathcal{A}_{n}=\int_{0}^{\infty} d t\left\langle B, \mathbf{x}\left|e^{-2 \pi t \alpha^{\prime} p^{+}\left(P^{-}-i \partial / \partial x^{+}\right)} V_{1} \ldots V_{n}\right| B, \mathbf{y}\right\rangle
$$

In particular, by inserting the boost operator $e^{V_{B}}$, where ${ }^{5}$

$$
V_{B}=v_{i} \oint_{\tau=0} d \sigma\left(X^{[1} \partial_{\sigma} X^{i]}+\frac{1}{2} S \gamma^{1 i} S\right)
$$

one recovers the eikonal scattering between moving branes performed in [14]. Dp-branes correspond however to solitonic BPS saturated solutions of Type IIA(B) supergravity, which preserve one half of the supersymmetries. The remaining half is realized on a short-multiplet containing 256 p-brane configurations; all the various components of the short-multiplet are related by supersymmetry transformations generated by the 16 broken supercharges. From this perspective, the original computation by Polchinski [1] or the eikonal scattering [14] correspond simply to the leading interaction between two generic components of the super-multiplet, that does not depend on the particular polarization of both states [4]. In order to get the spin-dependent part of D-brane interactions, one has to insert broken supercharges into the amplitude (6). In particular the correlator that will encode the eikonal scattering of two moving D-branes, including all spin effects, is the following:

$$
\mathcal{V}=\frac{1}{2} \int_{0}^{\infty} d t\left\langle B, \mathbf{x}=0\left|e^{-2 \pi t \alpha^{\prime} p^{+}\left(P^{-}-i \partial / \partial x^{+}\right)} e^{V_{B}} e^{\left(\eta Q_{-}+\widetilde{\eta} \widetilde{Q}_{-}\right)}\right| B, \mathbf{y}=\mathbf{b}\right\rangle
$$

$Q_{-}, \widetilde{Q}_{-}$being the $\mathrm{SO}(8)$ supercharges broken by the presence of D-branes (with $\eta, \widetilde{\eta}$ the corresponding supersymmetry parameters) and $V_{B}$ the boost operator (9) given above. Although the full computation of (10) will be extremely complicated, we will see that the leading interaction terms in (10) can be easily extracted. Since a configuration of parallel branes preserves $1 / 2$ of the supercharges, in light-cone gauge this implies that among the 16 linearly realized supercharges $S_{0}^{a}, \widetilde{S}_{0}^{\dot{a}}$, eight of them are left unbroken. Equations (6) and (10) require then the insertion of at least eight zero modes (that, due to the constraints (2), can be always chosen to be $S_{0}^{a}$ ) in order to get a non-vanishing result. This is better seen in a path-integral approach: in this case we will have an action admitting eight zero modes $S_{0}^{a}$; unless we do not insert vertex operators that soak up these zero modes, the Grassmanian integration over them will always make the amplitude vanishing.

This is indeed a very easy way to understand why the interaction between parallel moving branes start with the fourth power of velocity; since $V_{B}$ can provide at most two zero modes $S_{0}^{a}$, the first non-vanishing correlator has to contain four $V_{B}$ 's. In this particular case, moreover, the only role of the vertex operators is to provide these fermionic zero modes. This implies that all the massive string contributions, being

\footnotetext{
${ }^{5}$ The direction 1 entering in (9) will be Wick rotated to give the time direction.
} 
unaffected by the insertion of the vertex operators, will precisely cancel, exactly as in the evaluation of the static force (7). These amplitudes are therefore scale invariant, in the sense that their dependence on the D-brane distance $\mathbf{b}$ is exact, keeping the same functional form at any distance. The same argument implies, of course, that all the terms in (10) involving a maximum of eight fermion fields $S^{a}$ will be scale invariant and fixed by zero modes. Since each pair of supercharges in (10) provides two zero modes $S_{0}^{a}$ and an extra power of transfer momentum $q$, the leading effective potential between two moving D-branes will have the following schematic form, in configuration space:

$$
\Gamma \sim \frac{v^{4}}{r^{7-p}}+\frac{\theta^{2} v^{3}}{r^{8-p}}+\frac{\theta^{4} v^{2}}{r^{9-p}}+\frac{\theta^{6} v}{r^{10-p}}+\frac{\theta^{8}}{r^{11-p}}
$$

where $\theta=(\eta, \widetilde{\eta})$. This is indeed the form expected for spin-dependent interactions between D0-particles [2]. In this last case, by expanding (10) and performing all the algebra, one finds (normalizing to one the $v^{4}$ term and setting $\alpha^{\prime}=1$ )

$$
\begin{aligned}
\Gamma_{(1)}(\mathbf{v}, \mathbf{b}, \theta)=[ & v^{4}+2 i v^{2} v_{m}\left(\theta \gamma^{m n} \theta\right) \partial_{n}-2 v_{p} v_{q}\left(\theta \gamma^{p m} \theta\right)\left(\theta \gamma^{q n} \theta\right) \partial_{m} \partial_{n} \\
& -\frac{4 i}{9} v_{i}\left(\theta \gamma^{i m} \theta\right)\left(\theta \gamma^{n l} \theta\right)\left(\theta \gamma^{p l} \theta\right) \partial_{m} \partial_{n} \partial_{p} \\
& \left.+\frac{2}{63}\left(\theta \gamma^{m l} \theta\right)\left(\theta \gamma^{n l} \theta\right)\left(\theta \gamma^{p k} \theta\right)\left(\theta \gamma^{q k} \theta\right) \partial_{m} \partial_{n} \partial_{p} \partial_{q}\right] \frac{1}{\mathbf{b}^{7}}
\end{aligned}
$$

This is the full one-loop leading potential between two parallel D0-branes, including their spin interactions; in writing (12) we neglect possible contact terms that are anyway not detectable in this configuration. Being fixed by the ground states of the GreenSchwarz string only, the potential above should be reproduced in particular by a oneloop matrix theory computation. Indeed, all the spin interactions computed up to now in matrix theory [8] are reproduced by (12).

\section{Super-graviton scattering in matrix theory}

In this section we will show how to compute scattering amplitudes in matrix theory by using the potential (12) above, the explicit asymptotic particle states found in [10] and the Lehmann, Symanzik and Zimmermann (LSZ) reduction formula applied to matrix theory $[11,12]$. As mentioned in the introduction, we will work in the $N=2$ sector of the theory.

The $N=2 \mathrm{U}(2)$ matrix theory Hamiltonian

$$
H=\frac{1}{2} P_{\mu}^{0} P_{\mu}^{0}+\left(\frac{1}{2} \mathbf{P}_{\mu} \cdot \mathbf{P}_{\mu}+\frac{1}{4}\left(\mathbf{X}_{\mu} \times \mathbf{X}_{\nu}\right)^{2}+\frac{i}{2} \mathbf{X}_{\mu} \cdot \boldsymbol{\theta} \gamma_{\mu} \times \boldsymbol{\theta}\right)
$$

is a sum of an interacting $\mathrm{SU}(2)$ part describing relative motions and a free $\mathrm{U}(1)$ piece pertaining to the centre of mass ${ }^{6}$. The model has a potential with flat directions along the Cartan directions $x_{\mu}$ and $\theta^{3}$, whereas the remaining degrees of freedom

\footnotetext{
${ }^{6} \mathrm{In}(13)$ a vector notation for the adjoint representation of $\mathrm{SU}(2)$ is used, $\mathbf{X}_{\mu}=\left(Y_{\mu}^{I}, x_{\mu}\right)$ and $\boldsymbol{\theta}=\left(\theta^{I}, \theta^{3}\right)(I=1,2$ and $\mu=1, \ldots, 9)$. In the following we will work in a gauge in which $Y_{9}^{I}=0$.
} 
are represented by supersymmetric harmonic oscillators $Y_{\mu}^{I}(\mu \neq 9)$ and $\theta^{I}$. Upon introducing a large gauge invariant distance $x=\left(\mathbf{X}_{9} \cdot \mathbf{X}_{9}\right)^{1 / 2}=x_{9}$ as the separation of a pair of particles, the Hamiltonian (13) was shown [10] to possess asymptotic two particle states of the form

$$
\left|p_{\mu}^{1}, \mathcal{H}^{1} ; p_{\mu}^{2}, \mathcal{H}^{2}\right\rangle=\left|0_{B}, 0_{F}\right\rangle \frac{1}{x_{9}} e^{i\left(p^{1}-p^{2}\right) \cdot x} e^{i\left(p_{1}+p_{2}\right) \cdot X^{0}}\left|\mathcal{H}^{1}\right\rangle_{\theta^{0}+\theta^{3}}\left|\mathcal{H}^{2}\right\rangle_{\theta^{0}-\theta^{3}}
$$

where $\left|0_{B}, 0_{F}\right\rangle$ is the ground state of the superharmonic oscillators and $p_{\mu}^{1,2}, \mathcal{H}^{1,2}$ are respectively the momenta and polarizations of the two particles. The polarization states above have been explicitly constructed in [10] where it has also been shown how they fit into the $\underline{44} \oplus \underline{84} \oplus \underline{128}$ representations of $\mathrm{SO}(9)$, corresponding respectively to the graviton, three-form tensor and gravitino states.

In this hamiltonian approach to matrix theory, once we know the form of the asymptotic particle states (14), it is straightforward to form S-matrix elements of the form $S_{f i}=\langle$ out $|\exp \{-i H T\}|$ in $\rangle$. The object of interest is then

$$
x_{\mu}^{\prime}\left\langle 0_{B}, 0_{F}|\exp \{-i H T\}| 0_{B}, 0_{F}\right\rangle_{x_{\mu}}=e^{i \Gamma\left(x_{\mu}^{\prime}, x_{\mu}, \theta^{3}\right)}
$$

where we have explicitly shown that the ground states $\left|0_{B}, 0_{F}\right\rangle$ depend on the particular vacuum expectation value given to the Cartan moduli $x_{\mu}$ and $x_{\mu}^{\prime}$. In general the effective potential $\Gamma$ in (15) can also have a dependence on the fermionic variable $\theta^{3}$, but not on the fermionic $\mathrm{U}(1)$ term $\theta^{0}$ that decouples from the interaction. The vacuum to vacuum transition amplitude (15) may be now represented as a path integral with appropriate boundary conditions for the cartan variables

$$
e^{i \Gamma\left(x_{\mu}, x_{\mu}^{\prime}, \theta^{3}\right)}=\int_{\mathbf{X}_{\mu}=\left(0,0, x_{\mu}\right), \boldsymbol{\theta}=\left(0,0, \theta^{3}\right)}^{\mathbf{X}_{\mu}=\left(0,0, x_{\mu}^{\prime}\right), \boldsymbol{\theta}=\left(0,0, \theta^{3}\right)} \mathcal{D}\left(\mathbf{X}_{\mu}, \mathbf{A}, \mathbf{b}, \mathbf{c}, \boldsymbol{\theta}\right) \exp \left(i \int_{-T / 2}^{T / 2} L_{\mathrm{SYM}}\right) .
$$

The Lagrangian $L_{\mathrm{SYM}}$ is that of a supersymmetric Yang-Mills quantum mechanics with appropriate gauge fixing to which end we have introduced ghosts $\mathbf{b}, \mathbf{c}$ and the (Lagrange multiplier) gauge field $\mathbf{A}$. The crucial observation is that one can now compute the path integral above in the gauge of one's choice; in particular it can be computed via an expansion about classical trajectories $X_{\mu}^{3}(t) \equiv x_{\mu}^{\mathrm{cl}}(t)=b_{\mu}+v_{\mu} t$ and constant $\theta^{3}(t)=\theta^{3}$ which yields the quoted boundary conditions through the identification $b_{\mu}=\left(x_{\mu}^{\prime}+x_{\mu}\right) / 2$ and $v_{\mu}=\left(x_{\mu}^{\prime}-x_{\mu}\right) / T$.

$\mathrm{Up}$ to an overall normalization $\mathcal{N}$, our LSZ reduction formula for matrix theory gives

$$
\begin{aligned}
S_{f i}= & \delta^{9}\left(k_{\mu}^{\prime}-k_{\mu}\right) e^{-i k_{\mu} k_{\mu} T / 2} \\
& \int d^{9} x^{\prime} d^{9} x \exp \left(-i w_{\mu} x_{\mu}^{\prime}+i u_{\mu} x_{\mu}\right)\left\langle\mathcal{H}^{3}\left|\left\langle\mathcal{H}^{4}\left|e^{i \Gamma\left(v_{\mu}, b_{\mu}, \theta^{3}\right)}\right| \mathcal{H}^{1}\right\rangle\right| \mathcal{H}^{2}\right\rangle
\end{aligned}
$$

The leading factor expresses momentum conservation for the centre of mass, where $k_{\mu}=p_{\mu}^{1}+p_{\mu}^{2}$ and $k_{\mu}^{\prime}=p_{\mu}^{3}+p_{\mu}^{4}$ for the in and outgoing particles, respectively, and similarly for the relative momenta $u_{\mu}=\left(p_{\mu}^{1}-p_{\mu}^{2}\right) / 2$ and $w_{\mu}=\left(p_{\mu}^{4}-p_{\mu}^{3}\right) / 2$.

In a loopwise expansion of the matrix theory path integral one finds $\Gamma\left(v_{\mu}, b_{\mu}, \theta^{3}\right)=$ $v_{\mu} v_{\mu} T / 2+\Gamma_{(1)}+\Gamma_{(2)}+\ldots$ of which we consider only the first two terms in order to 
compare our results with tree level supergravity. Inserting this expansion into (17) and changing variables $d^{9} x^{\prime} d^{9} x \rightarrow d^{9}(T v) d^{9} b$, the integral over $T v_{\mu}$ may be performed via stationary phase. Dropping the normalization and the overall centre of mass piece the $S$-matrix then reads

$$
S_{f i}=e^{-i[(u+w) / 2]^{2} T / 2} \int d^{9} b e^{-i q_{\mu} b_{\mu}}\left\langle\mathcal{H}^{3}\left|\left\langle\mathcal{H}^{4}\left|e^{i \Gamma_{(1)}\left(u_{\mu}+w_{\mu} / 2, b_{\mu}, \theta^{3}\right)}\right| \mathcal{H}^{1}\right\rangle\right| \mathcal{H}^{2}\right\rangle
$$

where $q_{\mu}=w_{\mu}-u_{\mu}$. It is important to note that in (18) the variables $\theta^{3}$ are operators $\left\{\theta_{\alpha}^{3}, \theta_{\beta}^{3}\right\}=\delta_{\alpha \beta}$ whose expectation between polarization states $|\mathcal{H}\rangle$ yields the spin dependence of the scattering amplitude. The loopwise expansion of the effective action should be valid for the eikonal regime, i.e. large impact parameter $b_{\mu}$ or small momentum transfer $q_{\mu}$. This is actually the same kinematical regime in which we can trust the potential (12).

We have now all the tools needed to compute t-poles of scattering amplitudes in matrix theory. Although one could in this way analyze arbitrary tree level processes we will consider here graviton-graviton scattering; by taking the quantum mechanical expectation value of (12) between the polarization states in (18) associated to gravitons, we get the following t-pole amplitude:

$$
\begin{aligned}
& \mathcal{A}=\frac{1}{q^{2}}\left\{\frac{1}{2}\left(h_{1} h_{4}\right)\left(h_{2} h_{3}\right) v^{4}+2\left[\left(q h_{3} h_{2} v\right)\left(h_{1} h_{4}\right)-\left(q h_{2} h_{3} v\right)\left(h_{1} h_{4}\right)\right] v^{2}\right. \\
& +\left(v h_{2} v\right)\left(q h_{3} q\right)\left(h_{1} h_{4}\right)+\left(v h_{3} v\right)\left(q h_{2} q\right)\left(h_{1} h_{4}\right)-2\left(q h_{2} v\right)\left(q h_{3} v\right)\left(h_{1} h_{4}\right) \\
& -2\left(q h_{1} h_{4} v\right)\left(q h_{3} h_{2} v\right)+\left(q h_{1} h_{4} v\right)\left(q h_{2} h_{3} v\right)+\left(q h_{4} h_{1} v\right)\left(q h_{3} h_{2} v\right) \\
& +\frac{1}{2}\left[\left(q h_{1} h_{4} h_{3} h_{2} q\right)-2\left(q h_{1} h_{4} h_{2} h_{3} q\right)+\left(q h_{4} h_{1} h_{2} h_{3} q\right)-2\left(q h_{2} h_{3} q\right)\left(h_{1} h_{4}\right)\right] v^{2} \\
& -\left(q h_{2} v\right)\left(q h_{3} q\right)\left(h_{1} h_{4}\right)+\left(q h_{2} q\right)\left(q h_{3} v\right)\left(h_{1} h_{4}\right)-\left(q h_{1} q\right)\left(q h_{2} h_{3} h_{4} v\right) \\
& +\left(q h_{1} q\right)\left(q h_{3} h_{2} h_{4} v\right)-\left(q h_{4} q\right)\left(q h_{2} h_{3} h_{1} v\right)+\left(q h_{4} q\right)\left(q h_{3} h_{2} h_{1} v\right) \\
& -\left(q h_{1} v\right)\left(q h_{4} h_{2} h_{3} q\right)+\left(q h_{1} v\right)\left(q h_{4} h_{3} h_{2} q\right)-\left(q h_{4} v\right)\left(q h_{1} h_{2} h_{3} q\right) \\
& +\left(q h_{4} v\right)\left(q h_{1} h_{3} h_{2} q\right)+\left(q h_{1} h_{4} q\right)\left(q h_{2} h_{3} v\right)-\left(q h_{1} h_{4} q\right)\left(q h_{3} h_{2} v\right) \\
& +\frac{1}{8}\left[\left(q h_{1} q\right)\left(q h_{2} q\right)\left(h_{3} h_{4}\right)+2\left(q h_{1} q\right)\left(q h_{4} q\right)\left(h_{2} h_{3}\right)+2\left(q h_{1} q\right)\left(q h_{3} q\right)\left(h_{2} h_{4}\right)\right. \\
& \left.+\left(q h_{3} q\right)\left(q h_{4} q\right)\left(h_{1} h_{2}\right)\right]+\frac{1}{2}\left[\left(q h_{1} q\right)\left(q h_{4} h_{2} h_{3} q\right)-\left(q h_{1} q\right)\left(q h_{2} h_{4} h_{3} q\right)\right. \\
& -\left(q h_{1} q\right)\left(q h_{4} h_{3} h_{2} q\right)-\left(q h_{4} q\right)\left(q h_{1} h_{2} h_{3} q\right)+\left(q h_{4} q\right)\left(q h_{1} h_{3} h_{2} q\right) \\
& \left.-\left(q h_{4} q\right)\left(q h_{2} h_{1} h_{3} q\right)\right]+\frac{1}{4}\left[\left(q h_{1} h_{3} q\right)\left(q h_{4} h_{2} q\right)+\left(q h_{1} h_{2} q\right)\left(q h_{4} h_{3} q\right)\right. \\
& \left.\left.+\left(q h_{1} h_{4} q\right)\left(q h_{2} h_{3} q\right)\right]\right\}+\left[h_{1} \longleftrightarrow h_{2}, h_{3} \longleftrightarrow h_{4}\right]
\end{aligned}
$$

where again we neglected all terms within the curly brackets proportional to $q^{2} \equiv q_{\mu} q_{\mu}$, i.e. those that cancel the $1 / q^{2}$ pole.

The result above has to be now compared with the t-pole of tree level gravitongraviton scattering in eleven dimensional supergravity (compactified on a circle). Luckily this computation already appeared in the literature where it has been shown to be 
dimension independent [15]. Since matrix theory is formulated in terms of on shell degrees of freedom only, namely transverse physical polarizations and euclidean ninemomenta, we have just to fix all the gauge freedom and take the appropriate kinematics. Going to light-cone variables, we take the case of vanishing $p^{-}$momentum exchange ${ }^{7}$, that corresponds to our matrix theory computation,

$$
\begin{array}{ll}
p_{M}^{1}=\left(-\frac{1}{2}\left(v_{\mu}-q_{\mu} / 2\right)^{2}, 1, v_{\mu}-q_{\mu} / 2\right) & p_{M}^{2}=\left(-\frac{1}{2}\left(v_{\mu}-q_{\mu} / 2\right)^{2}, 1,-v_{\mu}+q_{\mu} / 2\right) \\
p_{M}^{4}=\left(-\frac{1}{2}\left(v_{\mu}+q_{\mu} / 2\right)^{2}, 1, v_{\mu}+q_{\mu} / 2\right) & p_{M}^{3}=\left(-\frac{1}{2}\left(v_{\mu}+q_{\mu} / 2\right)^{2}, 1,-v_{\mu}-q_{\mu} / 2\right)
\end{array}
$$

where momenta are measured in units of the compactified radius, so that $p^{-}=1$. Note that the vectors $u_{\mu}$ and $w_{\mu}$ of (17) are simply $u_{\mu}=v_{\mu}-q_{\mu} / 2$ and $w_{\mu}=v_{\mu}+q_{\mu} / 2$. Polarizations and momenta are subject to the de Donder gauge $p_{N}^{i} h^{i}{ }^{N}-(1 / 2) p_{M}^{i} h^{i}{ }_{N}{ }^{N}=0$ (no sum on $i$ ). We reduce to physical polarizations by using the residual gauge freedom to set $h_{+M}^{i}=0$ and solve the de Donder gauge condition in terms of the transverse traceless polarizations $h_{\mu \nu}^{i}$ for which $h_{-M}^{i}=-p_{\nu}^{i} h_{\nu M}^{i}$.

Finally, by plugging the above states in the amplitude reported in [15] and taking the t-pole ${ }^{8}$ part of it, one finds precisely the matrix theory amplitude (19).

Although the agreement above might have been expected from the scale invariance of the potential (12), it is clear that these results establish a very precise correspondence between the two models. In particular they pose the basis to analyze and interpret higher order matrix theory amplitudes that would correspond to loop effects in supergravity or M-theory. It is clear that such tests will be fundamental to establish the range of validity of matrix theory.

\section{References}

1. Polchinski, J. (1995): Phys. Rev. Lett. 75, 4724-4727

2. Harvey, J.A. (1998): Nucl. Phys. Proc. Suppl. B68, 113-118

3. Green, M.B., Gutperle, M. (1996): Nucl. Phys. B476, 484-514

4. Morales, J.F., Scrucca, C.A., Serone, M. (1998): Phys. Lett. B417 233-239

5. Morales, J.F., Scrucca, C.A., Serone, M. (1998): Nucl. Phys. B534, 223-249

6. Green, M.B., Schwarz, J.H., Witten, E. (1987): Superstring theory: volume 2, Cambridge University Press

7. Banks, T., Fischler, W., Shenker, S., Susskind, L. (1997): Phys. Rev. D55, 5112-5128

8. Kraus, P. (1998): Phys. Lett. B419, 73-78; Barrio, M., Helling, R., Polhemus, G. (1998): JHEP 05,012; McArthur, I.N. (1998): Nucl. Phys. B534, 183-201

9. Banks, T. (1998): Nucl. Phys. Proc. Suppl. B67, 180-224

10. Plefka, J.C., Waldron, A. (1998): Nucl. Phys. B512, 460-484

11. Plefka, J.C., Serone, M., Waldron, A. (1998): Phys. Rev. Lett. 81, 2866-2869

12. Plefka, J.C., Serone, M., Waldron, A. (1998): JHEP 11, 010

13. Susskind, L. (1997): Another conjecture about M(atrix) theory, hep-th/9704080

14. Bachas, C. (1996): Phys. Lett. B374, 37-42

15. Sannan, S. (1986): Phys. Rev. D34, 1749-1758

${ }^{7}$ We denote $p_{ \pm}=p^{\mp}=\left(p^{10} \pm p^{0}\right) / \sqrt{2}$ and our metric convention is $\eta_{M N}=\operatorname{diag}(-,+\ldots,+)$. ${ }^{8} t=q_{\mu}^{2}=-2 p_{M}^{1} p_{4}^{M}$ in the above parametrization. 